\title{
Upper triangular operator matrices, asymptotic intertwining and Browder, Weyl theorems
}

\author{
Bhagwati P. Duggal' ${ }^{1}$ In Ho Jeon ${ }^{2}$ and In Hyoun $\mathrm{Kim}^{3 *}$
}

${ }^{*}$ Correspondence:

ihkim@incheon.ac.kr

${ }^{3}$ Department of Mathematics,

Incheon National University,

Incheon, 406-772, Korea

Full list of author information is

available at the end of the article

\section{Springer}

\begin{abstract}
Given a Banach space $\mathcal{X}$, let $M_{C} \in B(\mathcal{X} \oplus \mathcal{X})$ denote the upper triangular operator matrix $M_{C}=\left(\begin{array}{c}A C \\ 0 B\end{array}\right)$, and let $\delta_{A B} \in B(B(\mathcal{X}))$ denote the generalized derivation $\delta_{A B}(X)=A X-X B$. If $\lim _{n \rightarrow \infty}\left\|\delta_{A B}^{n}(C)\right\|^{\frac{1}{n}}=0$, then $\sigma_{X}\left(M_{C}\right)=\sigma_{X}\left(M_{0}\right)$, where $\sigma_{x}$ stands for the spectrum or a distinguished part thereof (but not the point spectrum); furthermore, if $R=R_{1} \oplus R_{2} \in B(\mathcal{X} \oplus \mathcal{X})$ is a Riesz operator which commutes with $M_{C_{\text {, }}}$ then $\sigma_{x}\left(M_{C}+R\right)=\sigma_{x}\left(M_{C}\right)$, where $\sigma_{x}$ stands for the Fredholm essential spectrum or a distinguished part thereof. These results are applied to prove the equivalence of Browder's ( $a$-Browder's) theorem for $M_{0}, M_{C}, M_{0}+R$ and $M_{C}+R$. Sufficient conditions for the equivalence of Weyl's (a-Weyl's) theorem are also considered.

MSC: Primary 47B40; 47A10; secondary 47B47; 47A11
\end{abstract}

Keywords: Banach space; asymptotically intertwined; SVEP; polaroid operator

\section{Introduction}

A Banach space operator $T \in B(\mathcal{X})$, the algebra of bounded linear transformations from a Banach space $\mathcal{X}$ into itself, satisfies Browder's theorem if the Browder spectrum $\sigma_{b}(T)$ of $T$ coincides with the Weyl spectrum $\sigma_{w}(T)$ of $T$; $T$ satisfies Weyl's theorem if the complement of $\sigma_{w}(T)$ in $\sigma(T)$ is the set $\Pi_{0}(T)$ of finite multiplicity isolated eigenvalues of $T$. Weyl's theorem implies Browder's theorem, but the converse is generally false (see [1-3]). Let $M_{0}$ and $M_{C} \in B(\mathcal{X} \oplus \mathcal{X})$ denote, respectively, the upper triangular operators $M_{0}=A \oplus B$ and $M_{C}=\left(\begin{array}{cc}A & C \\ 0 & B\end{array}\right)$ for some operators $A, C, B \in B(\mathcal{X})$. It is well known that $\sigma_{x}\left(M_{0}\right)=\sigma_{x}(A) \cup \sigma_{x}(B)=\sigma_{x}\left(M_{C}\right) \cup\left\{\sigma_{x}(A) \cap \sigma_{x}(B)\right\}$ for $\sigma_{x}=\sigma$ or $\sigma_{b}$, and $\sigma_{w}\left(M_{0}\right) \subseteq$ $\sigma_{w}(A) \cup \sigma_{w}(B)=\sigma_{w}\left(M_{C}\right) \cup\left\{\sigma_{w}(A) \cap \sigma_{w}(B)\right\}$. The problem of finding sufficient conditions ensuring the equality of the spectrum (and certain of its distinguished parts) of $M_{0}$ and $M_{C}$, along with the problem of finding sufficient conditions for $M_{0}$ satisfies Browder's theorem and/or Weyl's theorem to imply $M_{C}$ satisfies Browder's theorem and/or Weyl's theorem (and vice versa), has been considered by a number of authors in the recent past (see [3], and some of the references cited there). For example, if either $A^{* \prime}$ or $B$ has the single-valued extension property, SVEP for short, then $\sigma\left(M_{0}\right)=\sigma\left(M_{C}\right)=\sigma(A) \cup \sigma(B)$. Again, if $\sigma_{w}\left(M_{C}\right)=\sigma_{w}(A) \cup \sigma_{w}(B)$, then $\sigma\left(M_{0}\right)=\sigma\left(M_{C}\right)=\sigma(A) \cup \sigma(B)$ [3, Proposition 3.2] and $M_{0}$ satisfies Browder's theorem if and only if $M_{C}$ satisfies Browder's theorem [3, Theorem 4.8]; furthermore, in such a case, $M_{0}$ satisfies Weyl's theorem if and only if $M_{C}$ satisfies Weyl's theorem if and only if $\Pi_{0}\left(M_{0}\right)=\Pi_{0}\left(M_{C}\right)$ [3, Theorem 5.1]. The equal-

(c) 2013 Duggal et al.; licensee Springer. This is an Open Access article distributed under the terms of the Creative Commons Attribution License (http://creativecommons.org/licenses/by/2.0), which permits unrestricted use, distribution, and reproduction in any medium, provided the original work is properly cited. 
ity $\sigma_{w}\left(M_{C}\right)=\sigma_{w}(A) \cup \sigma_{w}(B)$ may be achieved in a number of ways: if either $A$ and $A^{*}$, or $A$ and $B$, or $A^{*}$ and $B^{*}$, or $B$ and $B^{*}$ have SVEP, then $\sigma_{w}\left(M_{C}\right)=\sigma_{w}(A) \cup \sigma_{w}(B)$ [3, Proposition 4.5]. In this paper we consider conditions of another kind, conditions which do not assume SVEP.

Given $S, T \in B(\mathcal{X}), S$ and $T$ are said to be asymptotically intertwined by $X \in B(\mathcal{X})$ if $\lim _{n \rightarrow \infty}\left\|\delta_{S T}^{n}(X)\right\|^{\frac{1}{n}}=0$. Here $\delta_{S T} \in B(B(\mathcal{X}))$ is the generalized derivation $\delta_{S T}(X)=S X-X T$ and $\delta_{S T}^{n}=\delta_{S T}\left(\delta_{S T}^{n-1}\right)$. Evidently, $S$ and $T$ asymptotically intertwined by $X$ does not imply $T$ and $S$ asymptotically intertwined by $X$. Furthermore, $S$ and $T$ asymptotically intertwined by $X$ does not imply $\sigma(S)=\sigma(T)$, not even $\sigma(S) \subseteq \sigma(T)$; see [4, Example 3.5.9]. However, as we shall see, if $A, B, C$ are as in the definition of $M_{C}$ above, then $A$ and $B$ asymptotically intertwined by $C$ implies the equality of the spectra, and many distinguished parts thereof to spectrum of $M_{0}$ and $M_{C}$. We prove in the following that if $\lim _{n \rightarrow \infty}\left\|\delta_{A B}^{n}(C)\right\|^{\frac{1}{n}}=0$, then $M_{C}$ satisfies Browder's theorem if and only if $M_{0}$ satisfies Browder's theorem. If, additionally, the isolated points of $\sigma\left(M_{0}\right)$ are poles of the resolvent of $M_{0}$, then $M_{c}$ satisfies Weyl's theorem if and only if $M_{0}$ satisfies Weyl's theorem. Extensions to $a$-Browder's theorem, $a$-Weyl's theorem and perturbations by Riesz operators are considered.

\section{Notation and complementary results}

For a bounded linear Banach space operator $S \in B(\mathcal{X})$, let $\sigma(S), \sigma_{p}(S), \sigma_{a}(S), \sigma_{s}(S)$ and iso $\sigma(S)$ denote, respectively, the spectrum, the point spectrum, the approximate point spectrum, the surjectivity spectrum and the isolated points of the spectrum of $S$. Let $\alpha(S)$ and $\beta(S)$ denote the nullity and the deficiency of $S$, defined by

$$
\alpha(S)=\operatorname{dim} S^{-1}(0) \quad \text { and } \quad \beta(S)=\operatorname{codim} S(\mathcal{X}) .
$$

If the range $S(\mathcal{X})$ of $S$ is closed and $\alpha(S)<\infty$ (resp. $\beta(S)<\infty$ ), then $S$ is called an upper semi-Fredholm (resp. a lower semi-Fredholm) operator. If $S \in B(\mathcal{X})$ is either upper or lower semi-Fredholm, $S$ is called a semi-Fredholm operator, and ind $(S)$, the index of $S$, is then defined by ind $(S)=\alpha(S)-\beta(S)$. If both $\alpha(S)$ and $\beta(S)$ are finite, then $S$ is a Fredholm operator. The ascent, denoted $\operatorname{asc}(S)$, and the descent, denoted $\operatorname{dsc}(S)$, of $S$ are given by

$$
\operatorname{asc}(S)=\inf \left\{n: S^{-n}(0)=S^{-(n+1)}(0)\right\}, \quad \operatorname{dsc}(S)=\inf \left\{n: S^{n}(\mathcal{X})=S^{n+1}(\mathcal{X})\right\}
$$

(where the infimum is taken over the set of non-negative integers); if no such integer $n$ exists, then $\operatorname{asc}(S)=\infty$, respectively $\operatorname{dsc}(S)=\infty$. Let

$$
\begin{aligned}
& \Phi_{+}(S)=\{\lambda \in C: S-\lambda \text { is upper semi-Fredholm }\}, \\
& \Phi_{-}(S)=\{\lambda \in C: S-\lambda \text { is lower semi-Fredholm }\}, \\
& \Phi(S)=\{\lambda \in C: S-\lambda \text { is Fredholm }\}, \\
& \sigma_{S F_{+}}(S)=\left\{\lambda \in \sigma_{a}(S): \lambda \notin \Phi_{+}(S)\right\}, \\
& \sigma_{S F_{-}}(S)=\left\{\lambda \in \sigma_{a}(S): \lambda \notin \Phi_{-}(S)\right\}, \\
& \sigma_{e}(S)=\{\lambda \in \sigma(S): \lambda \notin \Phi(S)\}, \\
& \sigma_{w}(S)=\left\{\lambda \in \sigma(S): \lambda \in \sigma_{e}(S) \text { or } \operatorname{ind}(S-\lambda) \neq 0\right\},
\end{aligned}
$$




$$
\begin{aligned}
& \sigma_{a w}(S)=\left\{\lambda \in \sigma_{a}(S): \lambda \in \sigma_{S F_{+}}(S) \text { or } \operatorname{ind}(S-\lambda)>0\right\}, \\
& \sigma_{s w}(S)=\left\{\lambda \in \sigma_{s}(S): \lambda \in \sigma_{S F_{-}}(S) \text { or } \operatorname{ind}(S-\lambda)<0\right\}, \\
& \sigma_{b}(S)=\left\{\lambda \in \sigma(S): \lambda \in \sigma_{e}(S) \text { or } \operatorname{asc}(S-\lambda) \neq \operatorname{dsc}(S-\lambda)\right\}, \\
& \sigma_{a b}(S)=\left\{\lambda \in \sigma_{a}(S): \lambda \in \sigma_{S F_{+}}(S) \text { or } \operatorname{asc}(S-\lambda)=\infty\right\}, \\
& \sigma_{s b}(S)=\left\{\lambda \in \sigma_{s}(S): \lambda \in \sigma_{S F_{-}}(S) \text { or } \operatorname{dsc}(S-\lambda)=\infty\right\}, \\
& \Pi_{0}(S)=\left\{\lambda \in \operatorname{iso} \sigma(S): 0<\operatorname{dim}(S-\lambda)^{-1}(0)=\alpha(S-\lambda)<\infty\right\}, \\
& p_{0}(S)=\{\lambda \in \operatorname{iso} \sigma(S): \lambda \in \Phi(S), \operatorname{asc}(S-\lambda)=\operatorname{dsc}(S-\lambda)<\infty\}, \\
& H_{0}(S)=\left\{x \in \mathcal{X}: \lim _{n \rightarrow \infty}\left\|S^{n} x\right\|^{1 / n}=0\right\} .
\end{aligned}
$$

Here $\sigma_{w}(S)$ is the Weyl spectrum, $\sigma_{a w}(S)$ denotes the Weyl (essential) approximate point spectrum, $\sigma_{s w}(S)$ the Weyl (essential) surjectivity spectrum, $\sigma_{b}(S)$ the Browder spectrum, $\sigma_{a b}(S)$ the Browder (essential) approximate point spectrum, $\sigma_{s b}(S)$ the Browder (essential) surjectivity spectrum, and $H_{0}(S)$ the quasi-nilpotent part of $S$ [1]. Recall, [1], that $H_{0}(S)$ and $K(S)$, where $K(S)$ denotes the analytic core

$$
\begin{aligned}
K(S)= & \left\{x \in \mathcal{X} \text { : there exists a sequence }\left\{x_{n}\right\} \subset \mathcal{X} \text { and } \delta>0\right. \text { for which } \\
& \left.x=x_{0}, S\left(x_{n+1}\right)=x_{n} \text { and }\left\|x_{n}\right\| \leq \delta^{n}\|x\| \text { for all } n=1,2, \ldots\right\}
\end{aligned}
$$

are hyper-invariant (generally non-closed) subspaces of $S$ such that $S^{-p}(0) \subseteq H_{0}(S)$ for every integer $p \geq 0$ and $S K(S)=K(S)$. Recall also that if $0 \in$ iso $\sigma(S)$, then $\mathcal{X}=H_{0}(S) \oplus$ $K(S)$.

We say that $S$ has the single valued extension property, or SVEP, at $\lambda \in \mathrm{C}$ if for every open neighborhood $U$ of $\lambda$, the only analytic solution $f$ to the equation $(S-\mu) f(\mu)=0$ for all $\mu \in U$ is the constant function $f \equiv 0$; we say that $S$ has SVEP if $S$ has a SVEP at every $\lambda \in \mathrm{C}$. It is well known that finite ascent implies SVEP; also, an operator has SVEP at every isolated point of its spectrum (as well as at every isolated point of its approximate point spectrum).

$S \in B(\mathcal{X})$ satisfies Browder's theorem, shortened to $S$ satisfies Bt, if $\sigma_{w}(S)=\sigma_{b}(S)$ (if and only if $\sigma(S) \backslash \sigma_{w}(S)=p_{0}(S)$, see [1, p.156]); $S$ satisfies Weyl's theorem, shortened to $S$ satisfies Wt, if $\sigma(S) \backslash \sigma_{w}(S)=\Pi_{0}(S)$ (if and only if $S$ satisfies Bt and $p_{0}(S)=\Pi_{0}(S)$ ) [1, p.177]. The implication $\mathrm{Wt} \Longrightarrow \mathrm{Bt}$ is well known.

An isolated point $\lambda \in$ iso $\sigma(S)$ is a pole (of the resolvent) of $S \in B(\mathcal{X})$ if $\operatorname{asc}(S-\lambda)=$ $\operatorname{dsc}(S-\lambda)<\infty$. In such a case we say that $S$ is polar at $\lambda$; we say that $S$ is polaroid (resp., polaroid on a subset $F$ of the set of isolated points of $\sigma(S)$ ) if $S$ is polar at every $\lambda \in$ iso $\sigma(S)$ (resp., at every $\lambda \in F)$. Let $p(S)$ denote the set of poles of $S$.

Throughout the following, $M_{0} \in B(\mathcal{X} \oplus \mathcal{X})$ shall denote the diagonal operator $M_{0}=A \oplus$ $B$ and $M_{C} \in B(\mathcal{X} \oplus \mathcal{X})$ shall denote the upper triangular operator matrix $\left(\begin{array}{cc}A & C \\ 0 & B\end{array}\right)$, for some operators $A, B, C \in B(\mathcal{X})$. Recall, [5, Exercise 7, p.293], that $\operatorname{asc}(A) \leq \operatorname{asc}\left(M_{C}\right) \leq \operatorname{asc}(A)+$ $\operatorname{asc}(B)$ and $\operatorname{dsc}(B) \leq \operatorname{dsc}\left(M_{C}\right) \leq \operatorname{dsc}(A)+\operatorname{dsc}(B)$.

Lemma 2.1 If $\sigma\left(M_{0}\right)=\sigma\left(M_{C}\right)$, then $p\left(M_{0}\right)=p\left(M_{C}\right)$.

Proof Since $\sigma\left(M_{C}\right)=\sigma\left(M_{0}\right)=\sigma(A) \cup \sigma(B)$, if a complex number $\lambda \in p\left(M_{C}\right)$ or $p\left(M_{0}\right)$ then $\lambda \in \operatorname{iso}(\sigma(A) \cup \sigma(B))$. We consider the case in which $\lambda \in \operatorname{iso} \sigma(A) \cap$ iso $\sigma(B)$ : the argument 
works just as well for the case in which $\lambda \in \rho(A)(=C \backslash \sigma(A))$ or $\lambda \in \rho(B)$. Let $\lambda \in p\left(M_{C}\right)$. Then

$$
\operatorname{asc}(A-\lambda) \leq \operatorname{asc}\left(M_{C}-\lambda\right)<\infty \quad \text { and } \quad \operatorname{dsc}(B-\lambda) \leq \operatorname{dsc}\left(M_{C}-\lambda\right)<\infty .
$$

If $\lambda \in$ iso $\sigma(B)$ and $\operatorname{dsc}(B-\lambda)<\infty$, then $\operatorname{asc}(B-\lambda)=\operatorname{dsc}(B-\lambda)<\infty$ and $B$ is polar at $\lambda[1$, Theorem 3.81]. Now let $\lambda \in$ iso $\sigma(A)$. Since $M_{C}$ is polar at $\lambda, H_{0}\left(M_{C}-\lambda\right)=\left(M_{C}-\lambda\right)^{-p}(0)$ for some integer $p \geq 1$. Observe that

$$
H_{0}(A-\lambda)=H_{0}\left(M_{C}-\lambda\right) \cap \mathcal{X}=\left(M_{C}-\lambda\right)^{-p}(0) \cap \mathcal{X}=(A-\lambda)^{-p}(0) .
$$

Hence, if $\lambda \in$ iso $\sigma(A)$, then

$$
\begin{aligned}
\mathcal{X} & =H_{0}(A-\lambda) \oplus K(A-\lambda)=(A-\lambda)^{-p}(0) \oplus K(A-\lambda) \\
& \Longrightarrow \quad(A-\lambda)^{p} \mathcal{X}=0 \oplus(A-\lambda)^{p} K(A-\lambda)=K(A-\lambda) \\
& \Longrightarrow \quad \mathcal{X}=(A-\lambda)^{-p}(0) \oplus(A-\lambda)^{p} \mathcal{X},
\end{aligned}
$$

i.e., $A$ is polar at $\lambda$. Now, since

$$
\operatorname{asc}\left(M_{0}-\lambda\right) \leq \operatorname{asc}(A-\lambda)+\operatorname{asc}(B-\lambda) \quad \text { and } \quad \operatorname{dsc}\left(M_{0}-\lambda\right) \leq \operatorname{dsc}(A-\lambda)+\operatorname{dsc}(B-\lambda)
$$

we have

$$
\operatorname{asc}\left(M_{0}-\lambda\right)=\operatorname{dsc}\left(M_{0}-\lambda\right)<\infty
$$

i.e., $M_{0}$ is polar at $\lambda$. Conversely, if $\lambda \in p\left(M_{0}\right)$, then $\operatorname{asc}\left(M_{0}-\lambda\right)=\max \{\operatorname{asc}(A-\lambda), \operatorname{asc}(B-\lambda)\}$ and $\operatorname{dsc}\left(M_{0}-\lambda\right)=\max \{\operatorname{dsc}(A-\lambda), \operatorname{dsc}(B-\lambda)\}$ implies $\operatorname{asc}\left(M_{C}-\lambda\right) \leq \operatorname{asc}(A-\lambda)+\operatorname{asc}(B-\lambda)$ and $\operatorname{dsc}\left(M_{C}-\lambda\right) \leq \operatorname{dsc}(A-\lambda)+\operatorname{dsc}(B-\lambda)$ are both finite, hence equal. Thus $M_{C}$ is polar at $\lambda$.

Remark 2.2 A number of conditions guaranteeing (the spectral equality) $\sigma\left(M_{C}\right)=\sigma\left(M_{0}\right)$ are to be found in the literature. Thus, for example, if $A^{*}$ or $B$ has SVEP, or if $\sigma_{w}\left(M_{C}\right)=$ $\sigma_{w}(A) \cup \sigma_{w}(B)$, or $\sigma_{a w}\left(M_{C}\right)=\sigma_{a w}(A) \cup \sigma_{a w}(B)$ [3, (I) p.5 and Proposition 3.2], then $\sigma\left(M_{C}\right)=$ $\sigma\left(M_{0}\right)$. Compact operators have SVEP; hence, if either of $A$ or $B$ is compact, then $\sigma\left(M_{C}\right)=$ $\sigma\left(M_{0}\right)$.

Lemma 2.1 shows that if $B$ is a compact operator then $p\left(M_{0}\right)=p\left(M_{C}\right)$. A proof of the following lemma may be obtained from that of Lemma 2.1: we give here an independent proof, exploiting the additional information contained in the hypothesis.

Lemma 2.3 If $\sigma\left(M_{0}\right)=\sigma\left(M_{C}\right)$, then $p_{0}\left(M_{0}\right)=p_{0}\left(M_{c}\right)$.

Proof Once again we consider points $\lambda \in$ iso $\sigma(A) \cap$ iso $\sigma(B)$. Let $\lambda \in p_{0}\left(M_{C}\right)$. Then $\alpha\left(M_{C}-\right.$ $\lambda)=\beta\left(M_{C}-\lambda\right)<\infty$ implies $M_{C}-\lambda \in \Phi$, and this in turn implies $A-\lambda \in \Phi_{+}$and $B-\lambda \in \Phi_{-}$. Since $\lambda$ is isolated in $\sigma(A)$ and $\sigma(B), \lambda \in p_{0}(A) \cap p_{0}(B)$ [1, Theorem 3.77]. Consequently, $\lambda \in p\left(M_{0}\right)$; furthermore, since $\alpha\left(M_{0}-\lambda\right) \leq \alpha(A-\lambda)+\alpha(B-\lambda), \lambda \in p_{0}\left(M_{0}\right)$. Conversely, 
if $\lambda \in p_{0}\left(M_{0}\right)$, then $A-\lambda$ and $B-\lambda \in \Phi$, and hence (since $\lambda$ is isolated in $\sigma(A)$ and $\sigma(B)$ ) $\lambda \in p_{0}(A) \cap p_{0}(B)$. This, as above, implies $\lambda \in p_{0}\left(M_{C}\right)$.

The following technical lemma will be required in the sequel.

Lemma 2.4 If $A$ is polaroid on $\Pi_{0}\left(M_{C}\right)$ and $\sigma\left(M_{C}\right)=\sigma\left(M_{0}\right)$, then $\Pi_{0}\left(M_{C}\right) \subseteq \Pi_{0}\left(M_{0}\right)$.

Proof Evidently, $\left(M_{C}-\lambda\right)^{-1}(0) \neq \emptyset$ implies $\left(M_{0}-\lambda\right)^{-1}(0) \neq \emptyset$, and $\alpha\left(M_{C}-\lambda\right)<\infty$ implies $\alpha(A-\lambda)<\infty$. Let $\lambda \in \Pi_{0}\left(M_{C}\right)$; then $\lambda \in$ iso $\sigma\left(M_{0}\right)$. We prove that $\alpha(B-\lambda)<\infty$. Suppose to the contrary that $\alpha(B-\lambda)=\infty$. Since

$$
\left(M_{C}-\lambda\right)(x \oplus y)=\{(A-\lambda) x+C y\} \oplus(B-\lambda) y,
$$

either $\operatorname{dim}\left(C(B-\lambda)^{-1}(0)\right)<\infty$ or $\operatorname{dim}\left(C(B-\lambda)^{-1}(0)\right)=\infty$. If $\operatorname{dim}\left(C(B-\lambda)^{-1}(0)\right)<\infty$, then (since $\alpha(B-\lambda)=\infty)(B-\lambda)^{-1}(0)$ contains an orthonormal sequence $\left\{y_{j}\right\}$ such that $\left(M_{C}-\lambda\right)\left(0 \oplus y_{j}\right)=0$ for all $j=1,2, \ldots$. But then $\alpha\left(M_{C}-\lambda\right)=\infty$, a contradiction. Hence $\operatorname{dim}\left(C(B-\lambda)^{-1}(0)\right)=\infty$. Since $\lambda \in \rho(A) \cup$ iso $\sigma(A)$ and $A$ is (by hypothesis) polar at $\lambda$ (with, as observed above, $\alpha(A-\lambda)<\infty) \alpha(A-\lambda)=\beta(A-\lambda)<\infty$. Thus $\operatorname{dim}\left\{C(B-\lambda)^{-1}(0) \cap(A-\right.$ $\lambda) \mathcal{X}\}=\infty$, and so there exists a sequence $\left\{x_{j}\right\}$ such that $(A-\lambda) x_{j}=C y_{j}$ for all $j=1,2, \ldots$. But then $\left(M_{C}-\lambda\right)\left(x_{j} \oplus-y_{j}\right)=0$ for all $j=1,2, \ldots$, and hence $\alpha\left(M_{C}-\lambda\right)=\infty$. This contradiction implies that we must have $\alpha(B-\lambda)<\infty$. Since $\alpha\left(M_{0}-\lambda\right) \leq \alpha(A-\lambda)+\alpha(B-\lambda)$, we conclude that $\lambda \in \Pi_{0}\left(M_{0}\right)$.

Let $\delta_{S T} \in B(B(\mathcal{X}))$ denote the generalized derivation $\delta_{S T}(X)=S X-X T$, and define $\delta_{S T}^{n}$ by $\delta_{S T}^{n-1}\left(\delta_{S T}\right)$. The operators $S, T \in B(\mathcal{X})$ are said to be asymptotically intertwined by the identity operator $I \in B(\mathcal{X})$ if $\lim _{n \rightarrow \infty}\left\|\delta_{S T}^{n}(I)\right\|^{\frac{1}{n}}=0 ; S, T$ are said to be quasi-nilpotent equivalent if $\lim _{n \rightarrow \infty}\left\|\delta_{S T}^{n}(I)\right\|^{\frac{1}{n}}=\lim _{n \rightarrow \infty}\left\|\delta_{T S}^{n}(I)\right\|^{\frac{1}{n}}=0$ [4, p.253]. Quasi-nilpotent equivalence preserves a number of spectral properties [4, Proposition 3.4.11]. In particular:

Lemma 2.5 Quasi-nilpotent equivalent operators have the same spectrum, the same approximate point spectrum and the same surjectivity spectrum.

\section{Results}

Let $\mathcal{K}(\mathcal{X})$ denote the ideal of compact operators in $B(\mathcal{X})$. The following construction, known in the literature as the Sadovskii/Buoni, Harte and Wickstead construction [6, p.159], leads to a representation of the Calkin algebra $B(\mathcal{X}) / \mathcal{K}(\mathcal{X})$ as an algebra of operators on a suitable Banach space. Let $S \in B(\mathcal{X})$. Let $\ell^{\infty}(\mathcal{X})$ denote the Banach space of all bounded sequences $x=\left(x_{n}\right)_{n=1}^{\infty}$ of elements of $\mathcal{X}$ endowed with the norm $\|x\|_{\infty}:=$ $\sup _{n \in \mathbb{N}}\left\|x_{n}\right\|$, and write $S_{\infty}, S_{\infty} x:=\left(S x_{n}\right)_{n=1}^{\infty}$ for all $x=\left(x_{n}\right)_{n=1}^{\infty}$, for the operator induced by $S$ on $\ell^{\infty}(\mathcal{X})$. The set $m(\mathcal{X})$ of all precompact sequences of elements of $\mathcal{X}$ is a closed subspace of $\ell^{\infty}(\mathcal{X})$ which is invariant for $S_{\infty}$. Let $\mathcal{X}_{q}:=\ell^{\infty}(\mathcal{X}) / m(\mathcal{X})$, and denote by $S_{q}$ the operator $S_{\infty}$ on $\mathcal{X}_{q}$. The mapping $S \mapsto S_{q}$ is then a unital homomorphism from $B(\mathcal{X}) \rightarrow B\left(\mathcal{X}_{q}\right)$ with kernel $\mathcal{K}(\mathcal{X})$ which induces a norm decreasing monomorphism from $B(\mathcal{X}) / \mathcal{K}(\mathcal{X})$ to $B\left(\mathcal{X}_{q}\right)$ with the following properties (see [6, Section 17] for details):

(i) $S$ is upper semi-Fredholm, $S \in \Phi_{+}$, if and only if $S_{q}$ is injective, if and only if $S_{q}$ is bounded below;

(ii) $S$ is lower semi-Fredholm, $S \in \Phi_{-}$, if and only if $S_{q}$ is surjective;

(iii) $S$ is Fredholm, $S \in \Phi$, if and only if $S_{q}$ is invertible. 
Lemma 3.1 For every $S \in B(\mathcal{X}), \sigma_{e}(S)=\sigma\left(S_{q}\right), \sigma_{S F_{+}}(S)=\sigma_{a}\left(S_{q}\right)$ and $\sigma_{S F_{-}}(S)=\sigma_{s}\left(S_{q}\right)$.

Proof The following implications hold:

$$
\begin{aligned}
\lambda \notin \sigma_{S F_{+}}(S) & \Longleftrightarrow S-\lambda \in \Phi_{+} \quad \Longleftrightarrow(S-\lambda)_{q} \text { is bounded below } \\
& \Longleftrightarrow \lambda \notin \sigma_{a}\left(S_{q}\right), \\
\lambda \notin \sigma_{S F_{-}}(S) & \Longleftrightarrow S-\lambda \in \Phi_{-} \Longleftrightarrow(S-\lambda)_{q} \text { is onto } \\
& \Longleftrightarrow \lambda \notin \sigma_{S}\left(S_{q}\right) \text { and } \\
\lambda \notin \sigma_{e}(S) & \Longleftrightarrow S-\lambda \in \Phi \Longleftrightarrow(S-\lambda)_{q} \text { is invertible } \Longleftrightarrow \lambda \notin \sigma\left(S_{q}\right) .
\end{aligned}
$$

The following theorem is essentially known [7] we provide here an alternative proof, using quasi-nilpotent equivalence and the construction above. Let $\Sigma_{0}$ denote either of $\sigma_{e}$, $\sigma_{S F_{+}}, \sigma_{S F_{-}}, \sigma_{w}, \sigma_{a w}, \sigma_{s w}, \sigma_{b}, \sigma_{a b}$ and $\sigma_{s b}$.

Theorem 3.2 Let $S, R \in B(\mathcal{X})$. If $R$ is a Riesz operator which commutes with $S$, then $\sigma_{x}(S+$ $R)=\sigma_{x}(S)$, where $\sigma_{x} \in \Sigma_{0}$.

Proof It is clear from the definition of a Riesz operator $R \in B(\mathcal{X})$ that $R-\mu$ is Browder (i.e., $\mu \notin \sigma_{b}(R)$ ), and $a$-Browder and $s$-Browder, for all non-zero $\mu \in \sigma(R)$ (see, for example, [1, Theorem 3.111]). Hence $\sigma\left(R_{q}\right)=\{0\}$, i.e., $R_{q} \in B\left(\mathcal{X}_{q}\right)$ is quasi-nilpotent. Let $t \in[0,1]$; then $S$ commutes with $t R$ and $(S+t R)_{q}=S_{q}+t R_{q}$. It follows that

$$
\lim _{n \rightarrow \infty}\left\|\delta_{(S+t R)_{q} S_{q}}^{n}\left(I_{q}\right)\right\|^{\frac{1}{n}}=\lim _{n \rightarrow \infty}\left\|\delta_{S_{q}(S+t R)_{q}}^{n}\left(I_{q}\right)\right\|^{\frac{1}{n}}=0
$$

i.e., $S_{q}$ and $S_{q}+t R_{q}$ are quasi-nilpotent equivalent operators for all $t \in[0,1]$. Thus $\sigma_{x}((S+$ $\left.R)_{q}\right)=\sigma_{x}\left(S_{q}\right)$, where $\sigma_{x}=\sigma$ or $\sigma_{a}$ or $\sigma_{s}$. Hence

$$
\sigma_{x}(S+R)=\sigma_{x}(S) ; \quad \sigma_{x}=\sigma_{e} \text { or } \sigma_{a e} \text { or } \sigma_{s e}
$$

The semi-Fredholm index being a continuous function, we also have from the above that

$$
\sigma_{x}(S+R)=\sigma_{x}(S) ; \quad \sigma_{x}=\sigma_{w} \text { or } \sigma_{a w} \text { or } \sigma_{s w}
$$

To complete the proof, we prove next that $\sigma_{b}(S+R)=\sigma_{b}(S)$; the proof for $\sigma_{a b}$ and $\sigma_{s b}$ is similar, and left to the reader. It would suffice to prove that $0 \in \sigma_{b}(S) \Longleftrightarrow 0 \in \sigma_{b}(S+R)$. Suppose that $0 \notin \sigma_{b}(S)$. Then $S \in \Phi$ (and $\operatorname{asc}(S)=\operatorname{dsc}(S)<\infty$ ), hence $S+t R \in \Phi$ for all $t \in[0,1]$. For an operator $T$, let $\overline{\mathcal{N}^{\infty}(T)}$ and $T^{\infty}(\mathcal{X})$ denote, respectively, the closure of the hyper kernel and the hyper range of $T$. Then $\overline{\mathcal{N}^{\infty}(S+t R)} \cap(S+t R)^{\infty}(\mathcal{X})$ is constant on $[0,1]$, and so, since $\overline{\mathcal{N} \infty}(S) \cap S^{\infty}(\mathcal{X})=\mathcal{N}^{\infty}(S) \cap S^{\infty}(\mathcal{X})=\{0\}$, it follows that $\mathcal{N}^{\infty}(S+R) \cap$ $(S+R)^{\infty}(\mathcal{X})=\{0\}$. Consequently, $S+R$ has SVEP at 0 [1, Corollary 2.26]. But then since $S+R \in \Phi, S+R$ is Browder. Considering $S=(S+R)-R$ proves $0 \notin \sigma_{b}(S+R) \Longrightarrow 0 \notin \sigma_{b}(S)$.

The following lemma appears in [8, Lemma 2.3]. Let $\Pi_{0 f}(S)=\{\lambda \in \operatorname{iso} \sigma(S): \alpha(S-\lambda)<$ $\infty$. Clearly, $\Pi_{0}(S) \subseteq \Pi_{0 f}(S)$. 
Lemma 3.3 If $S, R \in B(\mathcal{X})$, and $R$ is a Riesz operator which commutes with $S$, then $\Pi_{0 f}(S+$ $R) \cap \sigma(S) \subseteq$ iso $\sigma(S)$.

Let $\Sigma=\Sigma_{0} \cup \sigma \cup \sigma_{a} \cup \sigma_{s}$.

Theorem 3.4 If $\lim _{n \rightarrow \infty}\left\|\delta_{A B}^{n}(C)\right\|^{\frac{1}{n}}=0$, then $\sigma_{x}\left(M_{C}\right)=\sigma_{x}\left(M_{0}\right)$, where $\sigma_{x} \in \Sigma$.

Proof A straightforward calculation shows that

$$
\delta_{M_{C} M_{0}}^{n}(I)=-\delta_{M_{0} M_{C}}^{n}(I)=\left(\begin{array}{cc}
0 & \delta_{A B}^{n-1}(C) \\
0 & 0
\end{array}\right) .
$$

Hence

$$
\lim _{n \rightarrow \infty}\left\|\delta_{M_{C} M_{0}}^{n}(I)\right\|^{\frac{1}{n}}=\lim _{n \rightarrow \infty}\left\|\delta_{M_{0} M_{C}}^{n}(I)\right\|^{\frac{1}{n}} \leq \lim _{n \rightarrow \infty}\left\|\delta_{A B}^{n-1}(C)\right\|^{\frac{1}{n}}=0
$$

i.e., $M_{C}$ and $M_{0}$ are quasi-nilpotent equivalent. Similarly, writing $M_{C(q)}$ for $\left(M_{C}\right)_{q}$ and $M_{0(q)}$ for $\left(M_{0}\right)_{q}$,

$$
\begin{aligned}
\lim _{n \rightarrow \infty}\left\|\delta_{M_{C(q)}}^{n} M_{0(q)}\left(I_{q}\right)\right\|^{\frac{1}{n}} & =\lim _{n \rightarrow \infty}\left\|\delta_{M_{0(q)} M_{C(q)}}^{n}\left(I_{q}\right)\right\|^{\frac{1}{n}} \\
& \leq \lim _{n \rightarrow \infty}\left\|\delta_{A_{q} B_{q}}^{n-1}\left(C_{q}\right)\right\|^{\frac{1}{n}} \\
& =\lim _{n \rightarrow \infty}\left\|\delta_{A B}^{n-1}(C)\right\|^{\frac{1}{n}}=0,
\end{aligned}
$$

i.e., $M_{C(q)}$ and $M_{0(q)}$ are quasi-nilpotent equivalent (in $B\left((\mathcal{X} \oplus \mathcal{X})_{q}\right)$ ). Hence $\sigma_{x}\left(M_{C}\right)=$ $\sigma_{x}\left(M_{0}\right)$, where $\sigma_{x}=\sigma$ or $\sigma_{a}$ or $\sigma_{s}$ or $\sigma_{e}$ or $\sigma_{S F_{+}}$or $\sigma_{S F_{-}}$. Since

$$
M_{0}=\left(\begin{array}{cc}
A & 0 \\
0 & I
\end{array}\right)\left(\begin{array}{ll}
I & 0 \\
0 & B
\end{array}\right)=\left(\begin{array}{ll}
I & 0 \\
0 & B
\end{array}\right)\left(\begin{array}{cc}
A & 0 \\
0 & I
\end{array}\right)
$$

and

$$
M_{C}=\left(\begin{array}{ll}
I & 0 \\
0 & B
\end{array}\right)\left(\begin{array}{ll}
I & C \\
0 & I
\end{array}\right)\left(\begin{array}{ll}
A & 0 \\
0 & I
\end{array}\right),
$$

where $\left(\begin{array}{ll}I & C \\ 0 & I\end{array}\right)$ is invertible, and since $\lambda \notin \sigma_{e}\left(M_{C}\right) \Longleftrightarrow \lambda \notin \sigma_{e}\left(M_{0}\right) \Longrightarrow A-\lambda, B-\lambda \in \Phi$ (similarly, $\lambda \notin \sigma_{S F_{+}}\left(M_{C}\right) \Longrightarrow A-\lambda, B-\lambda \in \Phi_{+}$and $\lambda \notin \sigma_{S F_{-}}\left(M_{C}\right) \Longrightarrow A-\lambda, B-\lambda \in \Phi_{-}$), $\operatorname{ind}\left(M_{C}-\lambda\right)=\operatorname{ind}(A-\lambda)+\operatorname{ind}(B-\lambda)=\operatorname{ind}\left(M_{0}-\lambda\right)$. Hence $\sigma_{x}\left(M_{C}\right)=\sigma_{x}\left(M_{0}\right)$, where $\sigma_{x}=\sigma_{w}$ or $\sigma_{a w}$ or $\sigma_{s w}$. Observe that

$$
\begin{aligned}
\sigma_{b}\left(M_{C}\right) & =\left\{\lambda \in \sigma\left(M_{C}\right): \lambda \in \sigma_{w}\left(M_{C}\right) \text { or } \lambda \notin \operatorname{iso} \sigma\left(M_{C}\right)\right\} \\
& =\left\{\lambda \in \sigma\left(M_{0}\right): \lambda \in \sigma_{w}\left(M_{0}\right) \text { or } \lambda \notin \operatorname{iso} \sigma\left(M_{0}\right)\right\}, \\
\sigma_{a b}\left(M_{C}\right) & =\left\{\lambda \in \sigma_{a}\left(M_{C}\right): \lambda \in \sigma_{a w}\left(M_{C}\right) \text { or } \lambda \notin \operatorname{iso} \sigma_{a}\left(M_{C}\right)\right\} \\
& =\left\{\lambda \in \sigma_{a}\left(M_{0}\right): \lambda \in \sigma_{a w}\left(M_{0}\right) \text { or } \lambda \notin \operatorname{iso} \sigma_{a}\left(M_{0}\right)\right\}
\end{aligned}
$$


and

$$
\begin{aligned}
\sigma_{s b}\left(M_{C}\right) & =\left\{\lambda \in \sigma_{s}\left(M_{C}\right): \lambda \in \sigma_{s w}\left(M_{C}\right) \text { or } \lambda \notin \operatorname{iso} \sigma_{s}\left(M_{C}\right)\right\} \\
& =\left\{\lambda \in \sigma_{s}\left(M_{0}\right): \lambda \in \sigma_{s w}\left(M_{0}\right) \text { or } \lambda \notin \operatorname{iso} \sigma_{s}\left(M_{0}\right)\right\}
\end{aligned}
$$

[1, Corollary 3.23, Theorem 3.23 and Theorem 3.27]. Hence $\sigma_{x}\left(M_{C}\right)=\sigma_{x}\left(M_{0}\right)$, where $\sigma_{x}=$ $\sigma_{b}$ or $\sigma_{a b}$ or $\sigma_{s b}$.

Remark 3.5 If $M \in B(\mathcal{X} \oplus \mathcal{X})$ is the operator $M=\left(\begin{array}{ll}A & C \\ D & B\end{array}\right)$ such that the entries $A, B, C$ and $D$ mutually commute, then $\sigma_{x}(M)=\left\{\lambda \in \mathrm{C}: 0 \in \sigma_{x}((A-\lambda)(B-\lambda)-C D)\right\}$ [9, Theorem 2.3], where $\sigma_{x}=\sigma$ or $\sigma_{e}$. Dispensing with the mutual commutativity hypothesis and assuming instead that $C D=D C=0, C$ commutes with $A$ and $B$, and $\lim _{n \rightarrow \infty}\left\|\delta_{A B}^{n}(D)\right\|^{\frac{1}{n}}=0$, an argument similar to that used to prove Theorem 3.4 shows that $\sigma_{x}(M)=\sigma_{x}\left(M_{C}\right)$, where $\sigma_{x}=\sigma$ or $\sigma_{a}$ or $\sigma_{s}$ or $\sigma_{e}$ or $\sigma_{S F_{ \pm}}$.

Theorem 3.6 Suppose that $\lim _{n \rightarrow \infty}\left\|\delta_{A B}^{n}(C)\right\|^{\frac{1}{n}}=0$. Then:

(a) $M_{C}$ satisfies Bt if and only if $M_{0}$ satisfies Bt.

(b) Let $R_{i} \in B(\mathcal{X}), i=1,2$, be Riesz operators such that $R=R_{1} \oplus R_{2}$ commutes with $M_{C}$. Then $M_{0}$ satisfies Bt $\Longleftrightarrow M_{C}+R$ satisfies Bt $\Longleftrightarrow M_{0}+R$ satisfies Bt $\Longleftrightarrow M_{C}$ satisfies Bt.

Proof The hypothesis $R$ commutes with $M_{C}$ implies $R$ commutes with $M_{0}, R_{1} C=C R_{2}$ and $\delta_{\left(M_{C}+R\right)\left(M_{0}+R\right)}^{n}(I)=\delta_{M_{C} M}^{n}(I)$.

(a) Recall that an operator $S$ satisfies Bt if and only if $\sigma_{w}(S)=\sigma_{b}(S)$. Hence the following implications hold:

$$
\begin{aligned}
M_{0} \text { satisfies Bt } & \Longleftrightarrow \sigma_{w}\left(M_{0}\right)=\sigma_{b}\left(M_{0}\right) \\
& \Longleftrightarrow \sigma_{w}\left(M_{c}\right)=\sigma_{b}\left(M_{C}\right) \quad \text { (Theorem 3.4) } \\
& \Longleftrightarrow M_{C} \text { satisfies Bt. }
\end{aligned}
$$

(b) The hypothesis $\lim _{n \rightarrow \infty}\left\|\delta_{A B}^{n}(C)\right\|^{\frac{1}{n}}=0$ implies that $M_{C}+R$ and $M_{0}+R$ are quasinilpotent equivalent $\left(\Longrightarrow\right.$ by Theorem 3.4 that $\sigma_{x}\left(M_{C}+R\right)=\sigma_{x}\left(M_{0}+R\right)$, where $\left.\sigma_{x} \in \Sigma\right)$. The operator $R$ being Riesz, Theorem 3.2 implies $\sigma_{x}(T+R)=\sigma_{x}(T)$, where $T=M_{C}$ or $M_{0}$ and $\sigma_{x}=\sigma_{w}$ or $\sigma_{b}$. The (two way) implications

$$
\begin{aligned}
M_{0} \text { satisfies Bt } & \Longleftrightarrow \sigma_{w}\left(M_{0}\right)=\sigma_{b}\left(M_{0}\right) \Longleftrightarrow \sigma_{w}\left(M_{0}+R\right)=\sigma_{b}\left(M_{0}+R\right) \\
& \left(\Longleftrightarrow M_{0}+R \text { satisfies Bt }\right) \\
& \Longleftrightarrow \sigma_{w}\left(M_{C}+R\right)=\sigma_{b}\left(M_{C}+R\right) \Longleftrightarrow M_{C}+R \text { satisfies Bt } \\
& \Longleftrightarrow \sigma_{w}\left(M_{C}\right)=\sigma_{b}\left(M_{C}\right) \Longleftrightarrow M_{C} \text { satisfies Bt }
\end{aligned}
$$

now complete the proof.

Remark 3.7 (i) $S \in B(\mathcal{X})$ satisfies $a$-Browder's theorem, $a$-Bt, if and only if $\sigma_{a w}(S)=\sigma_{a b}(S)$ (equivalently, if and only if $\sigma_{a}(S) \backslash \sigma_{a w}(S)=p_{0}^{a}(S)=\left\{\lambda \in\right.$ iso $\left.\sigma_{a}(S): S-\lambda \in \Phi_{+}\right\}=\left\{\lambda \in \sigma_{a}(S)\right.$ : 
$\left.S-\lambda \in \Phi_{+}, \operatorname{asc}(S-\lambda)<\infty\right][2$, Theorem 3.3]). Theorem 3.6 holds with Bt replaced by $a$-Bt. (Thus, if either $M_{0}$ or $M_{C}$ satisfies $a$-Bt, then $M_{0}, M_{C}, M_{0}+R$ and $M_{C}+R$ all satisfy $a-\mathrm{Bt}$.) Furthermore, since $S$ satisfies generalized Browder's theorem, gBt, if and only if it satisfies Bt and $S$ satisfies generalized $a$-Browder's theorem, $a$-gBt, if and only if it satisfies $a$-Bt [10], Bt may be replaced by gBt or $a$-gBt in Theorem 3.6. Here, we refer the interested reader to consult $[2,10]$ for information about gBt and $a$-gBt.

(ii) The equivalence $S$ satisfies Bt $\Longleftrightarrow S^{*}$ satisfies Bt is well known. This does not hold for $a$-Bt: $S$ satisfies $a$-Bt does not imply $S^{*}$ satisfies $a$-Bt (or vice versa). We say that $S$ satisfies $s$-Bt if $S^{*}$ satisfies $a$-Bt (equivalently, if $\sigma_{s b}(S)=\sigma_{s w}(S)$ ). It is easily seen, we leave the verification to the reader, if either $M_{0}$ or $M_{C}$ satisfies $s$-Bt, then (in Theorem 3.6) $M_{0}$, $M_{C}, M_{0}+R$ and $M_{C}+R$ all satisfy $s$-Bt.

We consider next a sufficient condition for the equivalence of Weyl's theorem for operators $M_{0}$ and $M_{C}$ such that $\lim _{n \rightarrow \infty}\left\|\delta_{A B}^{n}(C)\right\|^{\frac{1}{n}}=0$. We say in the following that an operator $S$ is finitely polaroid on a subset $F \subseteq$ iso $\sigma(S)$ if every $\lambda \in F$ is a finite rank pole of $S$. Evidently, $M_{0}$ is finitely polaroid if and only if $A$ and $B$ are finitely polaroid.

Theorem 3.8 Suppose that $\lim _{n \rightarrow \infty}\left\|\delta_{A B}^{n}(C)\right\|^{\frac{1}{n}}=0$.

(a) If $A$ is polaroid, then $M_{C}$ satisfies $W t$ if and only if $M_{0}$ satisfies $W t$.

(b) Let $R_{i} \in B(\mathcal{X}), i=1,2$, be Riesz operators such that $R=R_{1} \oplus R_{2}$ commutes with $M_{C}$. A sufficient condition for the equivalence $M_{C}+R$ satisfies $W t \Longleftrightarrow M_{0}+R$ satisfies Wt is that $M_{0}$ is finitely polaroid.

Proof (a) If $M_{C}$ satisfies Wt, then $\sigma\left(M_{C}\right) \backslash \sigma_{w}\left(M_{C}\right)=p_{0}\left(M_{C}\right)=\Pi_{0}\left(M_{C}\right)$. Since $\sigma\left(M_{0}\right)=$ $\sigma\left(M_{C}\right)$ and $\sigma_{w}\left(M_{C}\right)=\sigma_{w}\left(M_{0}\right)$ (Theorem 3.4) and since Wt implies Bt, Theorem 3.6(a) implies $\sigma\left(M_{0}\right) \backslash \sigma_{w}\left(M_{0}\right)=p_{0}\left(M_{0}\right) \subseteq \Pi_{0}\left(M_{0}\right)$. Consequently, $\Pi_{0}\left(M_{C}\right) \subseteq \Pi_{0}\left(M_{0}\right)$. Let $\lambda \in$ $\Pi_{0}\left(M_{0}\right)$. Then $\lambda \in$ iso $\sigma\left(M_{C}\right), \alpha(A-\lambda)<\infty$ and $\alpha(B-\lambda)<\infty$. Hence, since $\alpha(A-\lambda) \leq$ $\alpha\left(M_{C}-\lambda\right) \leq \alpha(A-\lambda)+\alpha(B-\lambda), \alpha\left(M_{C}-\lambda\right)<\infty$. Evidently, $\lambda \in$ iso $\sigma(A) \cup \rho(A)$. If $\lambda \in$ iso $\sigma(A)$, then $A$ polaroid implies $0<\alpha(A-\lambda)$, and hence $0<\alpha\left(M_{C}-\lambda\right)$. If instead $\lambda \in \rho(A)$, then $-(A-\lambda)^{-1} C x \oplus x \in\left(M_{C}-\lambda\right)^{-1}(0)$ for every $x \in(B-\lambda)^{-1}(0)$; once again, $0<\alpha\left(M_{C}-\lambda\right)$. Consequently, $\lambda \in \Pi_{0}\left(M_{C}-\lambda\right)=p_{0}\left(M_{C}-\lambda\right)=p_{0}\left(M_{0}-\lambda\right)$ and hence $\Pi_{0}\left(M_{0}\right)=p_{0}\left(M_{0}\right) \Longrightarrow$ $M_{0}$ satisfies Wt. Conversely, if $M_{0}$ satisfies Wt, then $\sigma\left(M_{C}\right) \backslash \sigma_{w}\left(M_{C}\right)=p_{0}\left(M_{C}\right)=p_{0}\left(M_{0}\right)=$ $\Pi_{0}\left(M_{0}\right)=\sigma\left(M_{0}\right) \backslash \sigma_{w}\left(M_{0}\right)$ and $\Pi_{0}\left(M_{0}\right) \subseteq \Pi_{0}\left(M_{C}\right)$. Since $A$ is polaroid (hence polar on $\left.\Pi_{0}\left(M_{C}\right)\right)$ and $\sigma\left(M_{0}\right)=\sigma\left(M_{C}\right)$, Lemma 2.4 implies $\Pi_{0}\left(M_{0}\right)=\Pi_{0}\left(M_{C}\right)$. Thus $M_{C}$ satisfies Wt.

(b) Start by observing that $\sigma\left(M_{0}\right)=\sigma\left(M_{C}\right)$, and hence $M_{C}$ is finitely polaroid if and only if $M_{0}$ is finitely polaroid (Lemma 2.3). Suppose $M_{0}+R$ satisfies Wt. Then the implication Wt $\Longrightarrow$ Bt combined with Theorem 3.6(b) implies that both $M_{0}+R$ and $M_{C}+R$ satisfy Bt. As noted in the proof of Theorem 3.6(b), $\sigma_{w}(T+R)=\sigma_{w}(T), T=M_{0}$ or $M_{C}$. Furthermore, since $M_{0}+R$ and $M_{C}+R$ are quasi-nilpotent equivalent, $\sigma_{x}\left(M_{0}+R\right)=\sigma_{x}\left(M_{C}+R\right), \sigma_{x}=$ $\sigma$ or $\sigma_{w}$ (Theorem 3.4). Hence

$$
\begin{aligned}
\Pi_{0}\left(M_{0}+R\right) & =\sigma\left(M_{0}+R\right) \backslash \sigma_{w}\left(M_{0}+R\right)=\sigma\left(M_{C}+R\right) \backslash \sigma_{w}\left(M_{C}+R\right) \\
& =p_{0}\left(M_{C}+R\right) \subseteq \Pi_{0}\left(M_{C}+R\right) .
\end{aligned}
$$

If $\lambda \in \Pi_{0}\left(M_{C}+R\right)$ and $\lambda \notin \sigma\left(M_{C}\right)$, then $\left(M_{C}-\lambda\right)$ is invertible and so $M_{C}-\lambda \in \Phi \Longrightarrow$ $M_{C}+R-\lambda \in \Phi$. Hence, since $\lambda \in$ iso $\sigma\left(M_{C}+R\right), \lambda \in p_{0}\left(M_{C}+R\right)$. If, instead, $\lambda \in \sigma\left(M_{C}\right)$, 
then $\lambda \in$ iso $\sigma\left(M_{C}\right)$ (Lemma 3.3) $\Longrightarrow \lambda \in$ iso $\sigma\left(M_{0}\right) \Longrightarrow \lambda \in p_{0}\left(M_{0}\right)$ (since $M_{0}$ is finitely polaroid) $\Longrightarrow \lambda \in p_{0}\left(M_{C}\right)$ (Lemma 2.3) $\Longrightarrow M_{C}-\lambda \in \Phi$, and this as above implies $\lambda \in$ $p_{0}\left(M_{c}+R\right)$. Hence $\Pi_{0}\left(M_{C}+R\right)=p_{0}\left(M_{C}+R\right)$, and $M_{C}+R$ satisfies Wt. The converse, $M_{C}+R$ satisfies $\mathrm{Wt} \Longrightarrow M_{0}+R$ satisfies Wt follows from a similar argument (recall that $M_{C}$ is finitely polaroid follows from the hypothesis that $M_{0}$ is finitely polaroid).

Remark 3.9 The equivalence of Theorem 3.8(b) extends to

$$
\begin{aligned}
M_{0} \text { satisfies Bt } & \Longleftrightarrow M_{0}+R \text { satisfies Wt } \Longleftrightarrow M_{C}+R \text { satisfies Wt } \\
& \Longleftrightarrow M_{C} \text { satisfies Bt. }
\end{aligned}
$$

This is seen as follows. The implication $M_{0}+R$ satisfies Wt $\Longrightarrow M_{0}$ satisfies Bt and $M_{C}+R$ satisfies Wt $\Longrightarrow M_{C}$ satisfies Bt are clear from Theorem 3.6(b). If $M_{0}$ satisfies Bt, then the hypothesis $M_{0}$ is finitely polaroid implies $M_{0}$ satisfies Wt. By Theorem 3.6(b), $M_{0}+R$ satisfies Bt, i.e., $\sigma\left(M_{0}+R\right) \backslash \sigma_{w}\left(M_{0}+R\right)=p_{0}\left(M_{0}+R\right) \subseteq \Pi_{0}\left(M_{0}+R\right)$. Let $\lambda \in \Pi_{0}\left(M_{0}+R\right)$. If $\lambda \notin$ $\sigma\left(M_{0}\right)$, then $\left(M_{0}-\lambda \in \Phi \Longrightarrow\right) M_{0}+R-\lambda \in \Phi \Longrightarrow \lambda \in p_{0}\left(M_{0}+R\right)$ (since $\lambda \in$ iso $\sigma\left(M_{0}+R\right)$ ); if $\lambda \in \sigma\left(M_{0}\right)$, then $\lambda \in$ iso $\sigma\left(M_{0}\right)$ (by Lemma 3.3) and so (since $M_{0}$ is finitely polaroid) $\lambda \in p_{0}\left(M_{0}\right) \Longrightarrow M_{0}-\lambda \in \Phi \Longrightarrow M_{0}+R-\lambda \in \Phi \Longrightarrow \lambda \in p_{0}\left(M_{0}+R\right)$. Thus, in either case, $\Pi_{0}\left(M_{0}+R\right) \subseteq p_{0}\left(M_{0}+R\right)$, and hence $M_{0}+R$ satisfies Wt. The proof for $M_{C}$ satisfies $\mathrm{Bt} \Longrightarrow M_{C}+R$ satisfies Wt is similar: recall from Lemma 2.3 that $M_{0}$ finitely polaroid implies $M_{C}$ finitely polaroid.

$a$-Wt. $T \in B(\mathcal{X})$ satisfies $a$-Weyl's theorem, $a$-Wt for short, if $T$ satisfies $a$-Bt and $p_{0}^{a}(T)=$ $\Pi_{0}^{a}(T)$ (equivalently, if $\sigma_{a}(T) \backslash \sigma_{a w}(T)=p_{0}^{a}(T)=\Pi_{0}^{a}(T)$ ), where $\Pi_{0}^{a}(T)=\left\{\lambda \in\right.$ iso $\sigma_{a}(T)$ : $0<\alpha(T-\lambda)<\infty$ ] [1]. We say in the following that $T$ is $a$-polaroid if $T$ is polar at every $\lambda \in$ iso $\sigma_{a}(T)$. Trivially, $a$-polaroid implies polaroid (indeed, $p_{0}^{a}(T)=p_{0}(T)$ in such a case), but the converse is not true in general. Theorem 3.8 has an $a$-Wt analogue, which we prove below. We note, however, that the perturbation of an operator by a commuting Riesz operator preserves neither its spectrum nor its approximate point spectrum: this will, per se, force us into making an assumption on the approximate point spectrum of $M_{0}$ and $M_{0}+R$ in the analogue of Theorem 3.8(b).

Theorem 3.10 Suppose that $\lim _{n \rightarrow \infty}\left\|\delta_{A B}^{n}(C)\right\|^{\frac{1}{n}}=0$.

(a) If $M_{0}$ is a-polaroid, then $M_{C}$ satisfies $a$-Wt if and only if $M_{0}$ satisfies $a$-Wt.

(b) Let $R_{i} \in B(\mathcal{X}), i=1,2$, be Riesz operators such that $R=R_{1} \oplus R_{2}$ commutes with $M_{C}$. If $\sigma_{a}\left(M_{0}\right)=\sigma_{a}\left(M_{0}+R\right)$, then a sufficient condition for the equivalence $M_{C}+R$ satisfies $a$-Wt $\Longleftrightarrow M_{0}+R$ satisfies $a$-Wt is that $M_{0}$ is finitely a-polaroid.

Proof (a) We prove $\Pi_{0}^{a}\left(M_{0}\right)=\Pi_{0}^{a}\left(M_{C}\right)$ : the proof of (a) would then follow from the fact that if $M_{0}$ satisfies $a$-Wt $\left(\Longrightarrow M_{0}\right.$ satisfies $a$-Bt $\Longleftrightarrow M_{C}$ satisfies $a$-Bt), then

$$
\Pi_{0}^{a}\left(M_{0}\right)=\sigma_{a}\left(M_{0}\right) \backslash \sigma_{a w}\left(M_{0}\right)=\sigma_{a}\left(M_{C}\right) \backslash \sigma_{a w}\left(M_{C}\right)=p_{0}^{a}\left(M_{C}\right) \subseteq \Pi_{0}^{a}\left(M_{C}\right)
$$

and if $M_{C}$ satisfies $a-\mathrm{Wt}$, then

$$
\Pi_{0}^{a}\left(M_{C}\right)=\sigma_{a}\left(M_{C}\right) \backslash \sigma_{a w}\left(M_{C}\right)=\sigma_{a}\left(M_{0}\right) \backslash \sigma_{a w}\left(M_{0}\right)=p_{0}^{a}\left(M_{0}\right) \subseteq \Pi_{0}^{a}\left(M_{0}\right)
$$


If $\lambda \in \Pi_{0}^{a}\left(M_{0}\right)$, then

$$
\begin{aligned}
& \lambda \in \text { iso } \sigma_{a}\left(M_{0}\right), \quad 0<\alpha\left(M_{0}-\lambda\right)<\infty \\
& \Longleftrightarrow \quad \lambda \in p_{0}\left(M_{0}\right) \quad\left(\text { since } M_{0} \text { is } a \text {-polaroid }\right) \\
& \Longleftrightarrow \quad \lambda \in\left(p_{0}(A) \cup p_{0}(B)\right) \cup\left(p_{0}(A) \cup \rho(B)\right) \cup\left(\rho(A) \cup p_{0}(B)\right) \\
& \Longrightarrow \quad \alpha\left(M_{C}-\lambda\right) \leq \alpha(A-\lambda)+\alpha(B-\lambda)<\infty, \\
& \quad \operatorname{asc}\left(M_{C}-\lambda\right) \leq \operatorname{asc}(A-\lambda)+\operatorname{asc}(B-\lambda)<\infty, \\
& \operatorname{dsc}\left(M_{C}-\lambda\right) \leq \operatorname{dsc}(A-\lambda)+\operatorname{dsc}(B-\lambda)<\infty \\
& \Longrightarrow \quad \operatorname{asc}\left(M_{C}-\lambda\right)=\operatorname{dsc}\left(M_{C}-\lambda\right)<\infty, \quad 0<\alpha\left(M_{C}-\lambda\right)<\infty \\
& \Longrightarrow \quad \lambda \in p_{0}\left(M_{C}\right) \subseteq \Pi_{0}\left(M_{C}\right) \subseteq \Pi_{0}^{a}\left(M_{C}\right)
\end{aligned}
$$

if instead $\lambda \in \Pi_{0}^{a}\left(M_{C}\right)$, then

$$
\begin{aligned}
\lambda & \in \operatorname{iso} \sigma_{a}\left(M_{C}\right), \quad 0<\alpha\left(M_{C}-\lambda\right)<\infty \\
& \Longleftrightarrow \quad \lambda \in \operatorname{iso} \sigma_{a}\left(M_{0}\right), \quad 0<\alpha\left(M_{C}-\lambda\right)<\infty \\
& \Longrightarrow \lambda \in p\left(M_{0}\right), \quad 0<\alpha\left(M_{C}-\lambda\right)<\infty \\
& \Longleftrightarrow \lambda \in p_{0}\left(M_{c}\right) \quad(\text { Lemma 2.4) } \\
& \Longleftrightarrow \lambda \in p_{0}\left(M_{0}\right) \quad(\text { Lemma 2.1) } \\
& \Longrightarrow \lambda \in \Pi_{0}\left(M_{0}\right) \subseteq \Pi_{0}^{a}\left(M_{C}\right) .
\end{aligned}
$$

(b) If $\sigma_{a}\left(M_{0}+R\right)=\sigma_{a}\left(M_{0}\right)$, then it follows from Lemma 2.4 and Theorem 3.4 that

$$
\sigma_{x}\left(M_{0}\right)=\sigma_{x}\left(M_{0}+R\right)=\sigma_{x}\left(M_{C}+R\right)=\sigma_{x}\left(M_{C}\right) ; \quad \sigma_{x}=\sigma_{a} \text { or } \sigma_{a w} .
$$

Recall from Remark 3.7 that if either of $M_{0}+R$ or $M_{C}+R$ satisfies $a$-Bt, then $M_{0}, M_{0}+R$, $M_{C}$ and $M_{C}+R$ all satisfy $a-B t$. Hence, in view of the spectral equalities above,

$$
p_{0}^{a}\left(M_{0}\right)=p_{0}^{a}\left(M_{C}\right)=p_{0}^{a}\left(M_{C}+R\right)=p_{0}^{a}\left(M_{0}+R\right),
$$

whenever either of $M_{0}, M_{0}+R, M_{C}$ and $M_{C}+R$ satisfies $a$-Bt. Observe that the hypothesis $M_{0}$ is finitely a-polaroid implies $p_{0}^{a}\left(M_{0}\right)=p_{0}\left(M_{0}\right)=p_{0}\left(M_{C}\right)=p_{0}^{a}\left(M_{0}+R\right)$; hence (since $\left.p_{0}^{a}\left(M_{0}\right)=p_{0}^{a}\left(M_{C}\right)=p_{0}^{a}\left(M_{C}+R\right)=p_{0}^{a}\left(M_{0}+R\right)\right) p_{0}^{a}(S)=p_{0}^{a}(T)$ for every choice of $S, T=M_{0}$ or $M_{C}$ or $M_{0}+R$ or $M_{C}+R$. We prove now that if either of $M_{0}+R$ and $M_{C}+R$ satisfies $a$-Wt, then $\Pi_{0}^{a}\left(M_{0}+R\right)=\Pi_{0}^{a}\left(M_{C}+R\right)$ : this would then imply that if one satisfies $a-\mathrm{Wt}$, then so does the other.

Suppose $M_{0}+R$ satisfies $a$-Wt. Then $p_{0}\left(M_{0}+R\right)=p_{0}^{a}\left(M_{0}+R\right)=\Pi_{0}^{a}\left(M_{0}+R\right)(\Longrightarrow$ $\left.\Pi_{0}^{a}\left(M_{0}+R\right)=\Pi_{0}\left(M_{0}+R\right)\right)$ and $\Pi_{0}^{a}\left(M_{0}+R\right) \subseteq \Pi_{0}^{a}\left(M_{C}+R\right)$. Let $\lambda \in \Pi_{0}^{a}\left(M_{c}+R\right)$; then $\lambda \in$ iso $\sigma_{a}\left(M_{C}+R\right)=$ iso $\sigma_{a}\left(M_{0}\right)$ implies $\lambda \in p_{0}\left(M_{0}\right)=p_{0}^{a}\left(M_{C}+R\right)$. Thus $\Pi_{0}^{a}\left(M_{C}+R\right) \subseteq$ $p_{0}^{a}\left(M_{C}+R\right)=p_{0}^{a}\left(M_{0}+R\right)=\Pi_{0}^{a}\left(M_{0}+R\right)$. Consequently, $\Pi_{0}^{a}\left(M_{0}+R\right)=\Pi_{0}^{a}\left(M_{C}+R\right)$ in this case. Suppose next that $M_{C}+R$ satisfies $a$-Wt. Then $p_{0}\left(M_{C}+R\right)=p_{0}^{a}\left(M_{C}+R\right)=\Pi_{0}^{a}\left(M_{C}+R\right)$ 
and $\Pi_{0}^{a}\left(M_{C}+R\right) \subseteq \Pi_{0}^{a}\left(M_{0}+R\right)$. Let $\lambda \in \Pi_{0}^{a}\left(M_{0}+R\right)$; then $\lambda \in$ iso $\sigma_{a}\left(M_{0}\right)$ implies $\lambda \in$ $p_{0}^{a}\left(M_{0}\right)=p_{0}^{a}\left(M_{C}+R\right)$. As above, this implies $\Pi_{0}^{a}\left(M_{0}+R\right)=\Pi_{0}^{a}\left(M_{C}+R\right)$.

The following corollary is immediate from Theorem 3.10(b).

Corollary 3.11 Suppose that $\lim _{n \rightarrow \infty}\left\|\delta_{A B}^{n}(C)\right\|^{\frac{1}{n}}=0$. If $R_{i} \in B(\mathcal{X}), i=1,2$, are quasinilpotent operators such that $R=R_{1} \oplus R_{2}$ commutes with $M_{C}$, then a sufficient condition for the equivalence $M_{C}+R$ satisfies $a-W t \Longleftrightarrow M_{0}+R$ satisfies $a$-Wt is that $M_{0}$ is finitely a-polaroid.

\section{Competing interests}

The authors declare that they have no competing interests.

Authors' contributions

All authors contributed equally.

\section{Author details}

18 Redwood Grove, Northfield Avenue, Ealing, London, W5 4SZ, United Kingdom. ²Department of Mathematics Education, Seoul National University of Education, Seoul, 137-742, Korea. ${ }^{3}$ Department of Mathematics, Incheon National University, Incheon, 406-772, Korea

\section{Authors' information}

Work carried out together whilst the first author was visiting Korea.

\section{Acknowledgements}

This work was supported by the Incheon National University Research Grant in 2012

Received: 8 February 2013 Accepted: 7 May 2013 Published: 29 May 2013

\section{References}

1. Aiena, P: Fredholm and Local Spectral Theory with Applications to Multipliers. Kluwer Academic, Dordrecht (2004)

2. Duggal, BP: SVEP, Browder and Weyl theorems. In: Jiménez, PMA, Bustamante, GJ, Djordjević, SV (eds.) Tópicas de Theoría de la Approximación. Textos Científicos BUAP Puebla, vol. III, pp. 107-146 (2009). http://www.fcfm.buap.mx/CA/analysis-mat/pdf/LIBRO_TOP_T_APPROX.pdf

3. Duggal, BP: Browder and Weyl spectra of upper triangular operator matrices. Filomat 24(2), 111-130 (2010). doi:10.2298/FIL1002111D

4. Laursen, KB, Neumann, MN: Introduction to Local Spectral Theory. Clarendon, Oxford (2000)

5. Taylor, AE, Lay, DC: Introduction to Functional Analysis. Wiley, New York (1980)

6. Müller, V: Spectral Theory of Linear Operators. Operator Theory Advances and Applications, vol. 139. Birkhäuser, Basel (2003)

7. Rakočević, V: Semi-Browder operators and perturbations. Stud. Math. 122, 131-137 (1997)

8. Oudghiri, M: Weyl's theorem and perturbations. Integral Equ. Oper. Theory 53, 535-545 (2005)

9. Harte, RE: Invertibility and singularity of operator matrices. Proc. R. Ir. Acad. A 88(2), 103-118 (1988)

10. Amouch, M, Zguitti, $\mathrm{H}$ : On the equivalence of Browder's and generalized Browder's theorem. Glasg. Math. J. 48, 179-185 (2006) 